\title{
The Application of Metal-Organic Frameworks as Anode Materials for Li-Ion Batteries
}

\author{
Ting-Wen ZHAO ${ }^{1, a, *}$, Zi-Geng LIU ${ }^{2, b}$, Kang-Li FU ${ }^{1, c}$, Yang $\mathrm{LI}^{1, \mathrm{~d}}$, Ming CAI ${ }^{1, \mathrm{e}}$ \\ ${ }^{1}$ Xi'an Thermal Power Research Institute Co., Ltd, Xi'an, 710054, PR China \\ ${ }^{2}$ Chemistry Department, University of Cambridge, Cambridge, CB2 1EW, U.K. \\ azhaotingwen@tpri.com.cn, ${ }^{\mathrm{b}} \mathrm{zl} 302 @$ cam.ac.uk, ${ }^{\mathrm{c}}$ fukangli@tpri.com.cn, \\ dliyangs@tpri.com.cn, ${ }^{\mathrm{e}}$ caiming@tpri.com.cn
}

Keywords: Metal-organic frameworks, Anode material, Li-ion batteries

\begin{abstract}
Metal-organic frameworks have drawn great attention over the last two decades owing to their interesting structures and potential applications in various areas such as gas storage, catalysis and energy storage. This porous crystalline material also has potential application in lithium-ion batteries as novel electrode materials. In this paper, a metal-organic framework $\mathrm{Mn}_{2}(\mathrm{OH})_{2}\left(\mathrm{C}_{4} \mathrm{O}_{4}\right)$ was synthesized with squaric acid through hydrothermal method. As an anode material for lithium-ion batteries, it has shown satisfying electrochemical properties in terms of specific capacity and cycling performance. Specifically, it exhibited a large reversible capacity of $c a .450 \mathrm{~m} \mathrm{Ah} \mathrm{g}^{-1}$ at a high current density of $400 \mathrm{~m} \mathrm{~A} \mathrm{~g}^{-1}$ after 50 cycles within the voltage range of $0.005-2 \mathrm{~V}$.
\end{abstract}

\section{Introduction}

The rechargeable lithium-ion battery (Li-ion battery or LIB), as one of the electrochemical energy conversion devices, has been widely applied in portable electronics such as mobile phones and laptops, and is expected to power electric vehicles and stationary power storage[1]. In general, graphite has been widely used as an anode in commercially available LIBs. However, the relatively low capacity and poor rate-performance of graphite makes it difficult to satisfy the high energy and power density requirements for LIBs used in large-scale energy storage [2]. As a result, there is an immediate need to develop novel anode materials with high capacity and longer cycling life [3].

Metal-organic frameworks (MOFs), a class of crystalline porous hybrid materials, have attracted tremendous attention over the last two decades. In addition to properties such as high porosity and massive internal surface area, the most striking feature of MOFs over traditional porous materials (such as polymers and zeolites)is their uniform yet tunable pore sizes [4,5]. These properties, together with the flexibility of metal ions and linkers that can be used, render MOFs as promising materials in a number of fields. These include applications in gas/liquid storage or separation; catalysis; biomedical applications, such as drug delivery; sensor materials; and energy storage [6-8].Recently, MOF materials have also attracted considerable interest in clean energy applications, such as fuel cells, super capacitors and LIBs [3]. Porous materials have been widely used in these areas owing to their large internal surface area and porosity. On account of this, MOFs have attracted great interest as electrode materials in the Li-ion batteries, as they may perform as active redox sites during electrochemical process [9-11].The first use of a MOF as anode in reversible lithium storage was reported by Chen et al. using $\mathrm{Zn}_{4} \mathrm{O}(\mathrm{BTB})_{2}\left(\mathrm{BTB}^{3-}=1,3,5\right.$-benzenetribenzoate). Electrochemical performance results showed that this material has a high irreversible capacity in the first cycle (425 and $110 \mathrm{mAhg}^{-1}$ for discharge and charge, respectively), and a lower reversible discharge-charge capacity in the following cycles [12]. Since then, attempts on exploring novel MOFs materials as electrode have been carried out.

In this paper, squarate based MOFs have been chosen because of the redox properties of both metal centres and the squarate dianion. Squarate contains a four-member cyclic aromatic structure which is characterized by electron delocalization over the carbon and oxygen atoms. In addition, squaric acid lig ands can theoretically accept two electrons per molecule and also act as a redox mediator to enhance the electron-transfer in the structure [13]. Herein, we demonstrated a 
convenient one-pot hydrothermal method to obtain a squarate-based MOF, $\mathrm{Mn}_{2}(\mathrm{OH})_{2}\left(\mathrm{C}_{4} \mathrm{O}_{4}\right)$ (referred as Mn-MOF), and explored the possible application of this materialas anodes in the LIBs as well as understanding its electrochemical performance.

\section{Experimental Section}

All chemicals were purchased from commercial suppliers and used as received. The materials were synthesized through the hydrothermal method. Squaric acid $(1 \mathrm{mmol}, 114 \mathrm{mg})$ and potassium hydroxide $(1.0 \mathrm{M}, 3 \mathrm{~mL})$ were added to a solution of metal salt $(1.5 \mathrm{mmol})$ in $\mathrm{H}_{2} \mathrm{O}(3.0 \mathrm{~mL})$. The solution was transferred into a $23 \mathrm{~mL}$ Teflon-lined autoclave. Then, the autoclave was capped tightly and placed in the oven at $200{ }^{\circ} \mathrm{Cfor} 15 \mathrm{~h}$. After cooling down to room temperature, crystalline products were filtered off, washed with distilled water $(3 \times)$. Finally, the materials were obtained after dired at ambient temperature. Elemental Analysis(\%) for $\mathrm{Mn}_{2} \mathrm{C}_{4} \mathrm{O}_{6} \mathrm{H}_{2}$ : C, 18.76; $\mathrm{H}$, 0.78; Found (\%): C, 18.68; H, 0.76.

The elemental analysis (EA) data for C, H were obtained from Perkin-Elmer 240 Elemental analyzer. The powder X-ray diffraction (PXRD) patterns were recorded on a PANalytical EMPYREAN X-ray diffract meter using $\mathrm{Cu} \mathrm{K \alpha}$ radiation $(\lambda=1.54 \AA)$.Samples were scanned in the $2 \theta$ range of $5^{\circ}$ to $90^{\circ}$. Infrared spectra were recorded by Perkin-Elmer Spectrum. Electrochemical tests were carried out using two-electrode (coin type) cells, which were assembled inside an argon-filled glove box. The working electrodes were prepared by mixing the MOF materials and carbon black (super P) at a weight ratio of 1:1. Glass fibre was used as a separator, and pure lithium foil was used as counter electrode. The electrolyte consisted of $1 \mathrm{M} \mathrm{LiPF}_{6}$ in ethylene carbonate (EC) and dimethyl carbonate (DMC) (1:1 by volume). Electrochemical performances tests were conducted at various current densities (100, 400 and1000 $\mathrm{m} \mathrm{Ag}^{-1}$, respectively) in a voltage range of 0.005 - 2 V and recorded on a Land BT2001A battery test system.

\section{Results and Discussion}

Synthesis and Characterization. Mn-MOF has been synthesized through a hydrothermal process with good purity and crystallinity. The diffraction peaks for Mn-MOF could be indexed to monoclinic system with space group $P 2_{1} / c$, and the lattice parameters were calculated as follows: a $=3.22730(10) \AA, \mathrm{b}=10.0328(4) \AA, \mathrm{c}=8.4979(3) \AA, \beta=99.461(2)^{\circ}, \mathrm{V}=271.410(17) \AA^{3}$. The composition of the material was determined by IR analysis. The IR spectrum of Mn-MOF is shown in Fig. 1. The sharp peak absorbed at $3480 \mathrm{~cm}^{-1}$ indicates that the $\mathrm{O}-\mathrm{H}$ groups only act as the bridging molecules, instead of involving in the hydrogen bonding interactions. The broad and strong band in the 1700-1400 $\mathrm{cm}^{-1}$ range can be assigned to a mixture of C-C $\left(1551 \mathrm{~cm}^{-1}\right)$ and C-O $\left(1485 \mathrm{~cm}^{-1}\right)$ stretching vibrations. The absence of a sharp peak in the region of 1700-1680 $\mathrm{cm}^{-1}$ supports the XRD data, indicating the complete deprotonation of the carboxyl groups and all the $\mathrm{C}-\mathrm{O}$ groups are coordinated to the metal atoms. In addition, the sharp peak at $1087 \mathrm{~cm}^{-1} \mathrm{can}$ be ascribed to the $\mathrm{C}-\mathrm{C}-\mathrm{O}$ stretching in the squarate. The purity of the compound was further confirmed by the elemental analysis. 


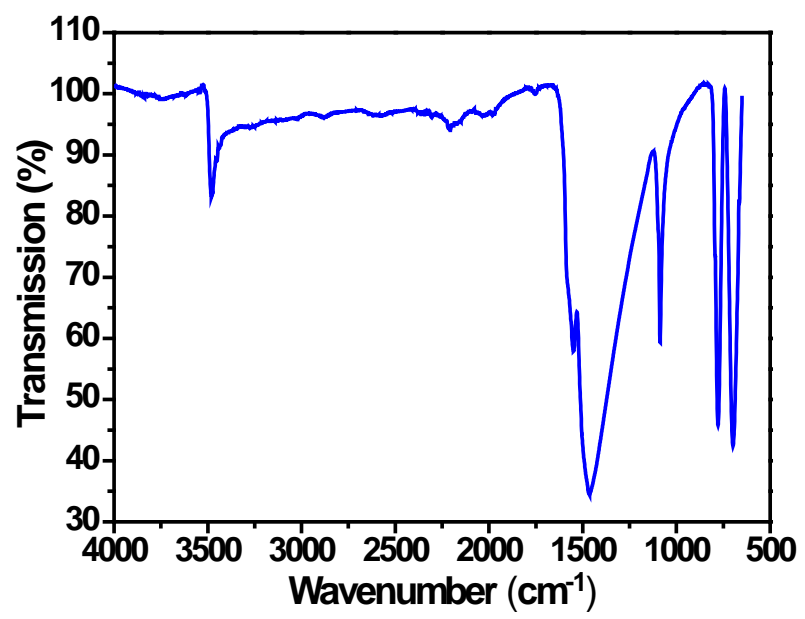

Fig. 1.FT-IR spectrum of Mn-MOF.

Electrochemical Performance Fig.2a shows the selected discharge/charge curves of the Mn-MOF, which was cycled at a constant current density of $100 \mathrm{mAg}^{-1}$ between a potential range of $0.005-2 \mathrm{~V}$ (vs. $\mathrm{Li} / \mathrm{Li}^{+}$). During the first discharge process, the potential of the electrode quickly fell to about $0.8 \mathrm{~V}$ with a small plateau, which was associated with the insertion of Li. An obvious long plateau was observed at $c a$. $0.55 \mathrm{~V}$ with a capacity of $944 \mathrm{mAh} \mathrm{g}^{-1}$ (theoretically consumption of 9.01 moles of Li per mole of Mn-MOF). Following those plateaus, the potential continuously decreased until the cut-off voltage $(0.005 \mathrm{~V})$, resulting in a capacity of $1625 \mathrm{mAh} \mathrm{g}^{-1}$. The first charge profile gradually increased to the upper cut-off voltage $(2 \mathrm{~V})$ with a sloping plateau at $c a .0 .9 \mathrm{~V}$. The overall charge capacity was found to be $763 \mathrm{mAh} \mathrm{g}^{-1}$.The large irreversible capacity in the initial cycle can be ascribed to the decomposition of the electrolyte at low potential to form a solid electrolyte interface (SEI) on the electrode [14,15]. In the second discharge cycle, the long voltage plateau at ca. $0.55 \mathrm{~V}$ disappeared, possibly due to the structural change for the electrode in the first cycle. The capacity values for the discharge and charge profiles in the second cycle were 788 and $622 \mathrm{mAh} \mathrm{g}^{-1}$, respectively. Upon comparing the capacity of the first and second discharge cycles, the capacity loss is around $48 \%$.

The plateau voltages in first three cycles are reflected as peaks in the differential capacity vs. voltage plots shown in Fig. 2b. The first cycle is substantially different from the subsequent cycles. During the first cycle, a small peak appears at $0.8 \mathrm{~V}$, followed by a sharp peak at $0.55 \mathrm{~V}$, indicating the two reduction steps. At the initial stage of lithium intercalation, a small amount of lithium can be inserted into the crystal structure of the as-prepared material. The sharp peak may corresponds to the complete reduction of $\mathrm{Mn}(\mathrm{II})$ and the formation of SEI. The oxidation peak at $1.00 \mathrm{~V}$ can be attributed to the delithiation of the electrode. In the subsequent cycles, the reduction peak at $0.80 \mathrm{~V}$ disappeared and the remaining peak slightly shifted towards higher potential with decreasing peak intensity. The difference between the first and the second curves is due to an irreversible phase transformation during the process of lithium insertion and extraction in the initial cycle. Apart from the first cycle, the subsequent cycles overlap well, indicating enhanced reversibility of the Mn-MOF during cycling.

The cycling performance and coulombic efficiency at varying current densities of 400 and 1000 $\mathrm{mA} \mathrm{g}{ }^{-1}$ areshown in Fig. 2c. For both conditions, the specific capacity of the Mn-MOF was stabilized after 20 cycles. At a current density of $400 \mathrm{~mA} \mathrm{~g}^{-1}$, the electrode exhibited a capacity decline in the first 20 cycles, while it remains a reversible lithium storage capacity up to $c a$. 450 $\mathrm{mAh} \mathrm{g}^{-1}$ afterwards. This exceeds the capacity of traditional graphite at a high current density within the same voltage range. The improved electrochemical performance can be ascribed to the porous hollow structure of Mn-MOF. The capacity even slightly increased after 50 cycles, which might be attributed to the improvement of the Li ion accessibility in the material. The columbic efficiency 
(defined as the ratio of charge capacity to discharge capacity) was found to be over $98 \%$ after 20 cycles. It is noted that columbic efficiency approached almost $100 \%$ for the sample discharged at a current density of $1000 \mathrm{~mA} \mathrm{~g}^{-1}$ after 20 cycles.
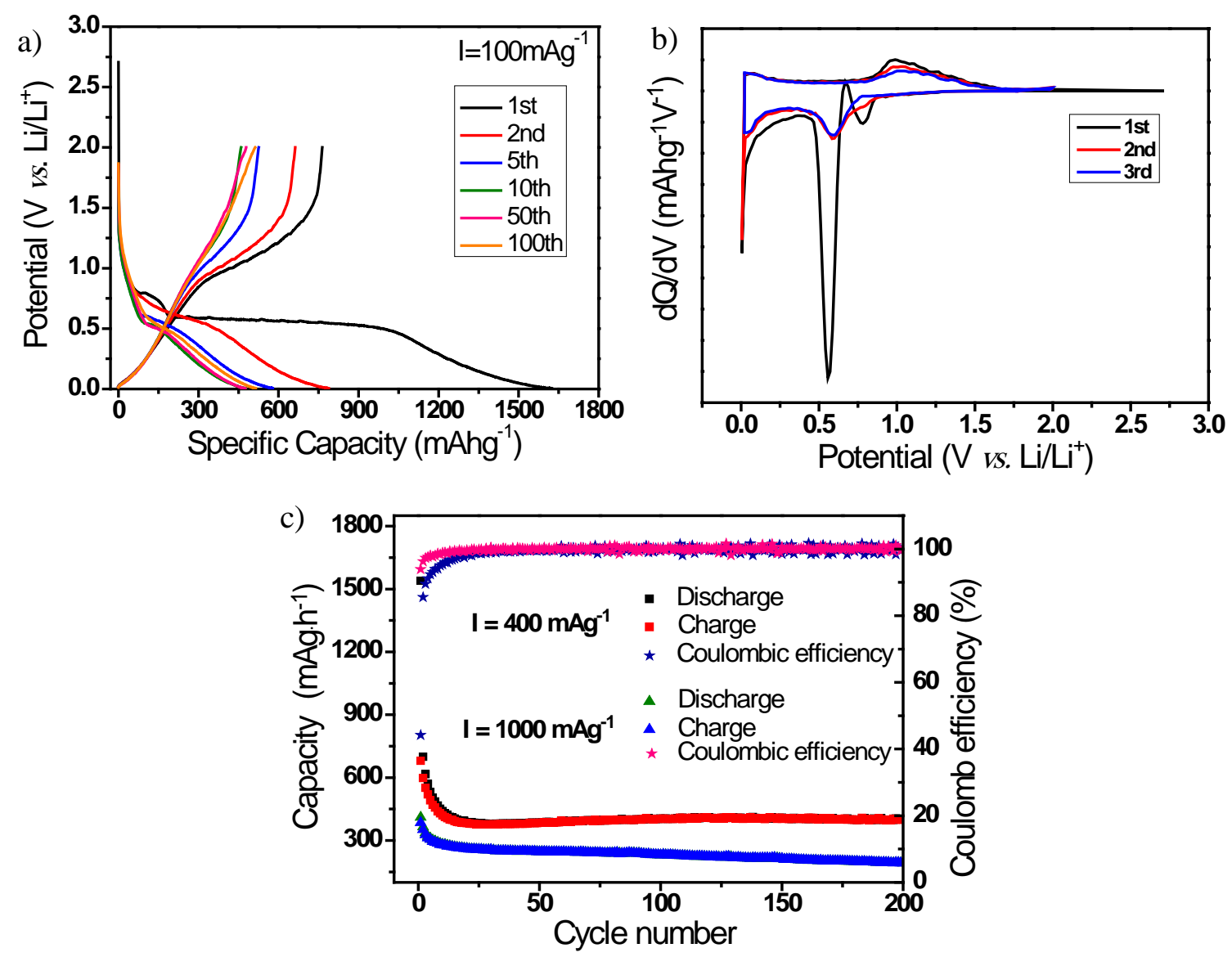

Fig. 2.a) selected discharge-charge curves at current density of $100 \mathrm{mAg}^{-1}$; b) first three cycle differential capacity, dQ/dV vs. voltage plots extracted from (a), c) cycling performance and coulombic efficiency at varying current densities of 400 and $1000 \mathrm{~mA} \mathrm{~g}^{-1}$.

To investigate the electrode composition and determine if any significant structural change soccurred during discharge-charge cycling, ex situ PXRD and FT-IR experiments were carried out. As shown in Fig. 3a, the majority of the diffraction peaks disappeared after cycling, which might be due to the collapse of the structure after the first discharge. FT-IR studies on the cycled electrodes were carried out to understand the electrode compositions (Fig. 3b). The presence of the characteristic squarate peaks in the region of $1480-1000 \mathrm{~cm}^{-1}$ of Mn-MOF in the bare electrode as well as in both discharged and charged electrodes illustrates that squarate dianionmay be reversibly involved in the cycling, although the peaks for the discharged and charged electrodes are slightly shifted. 


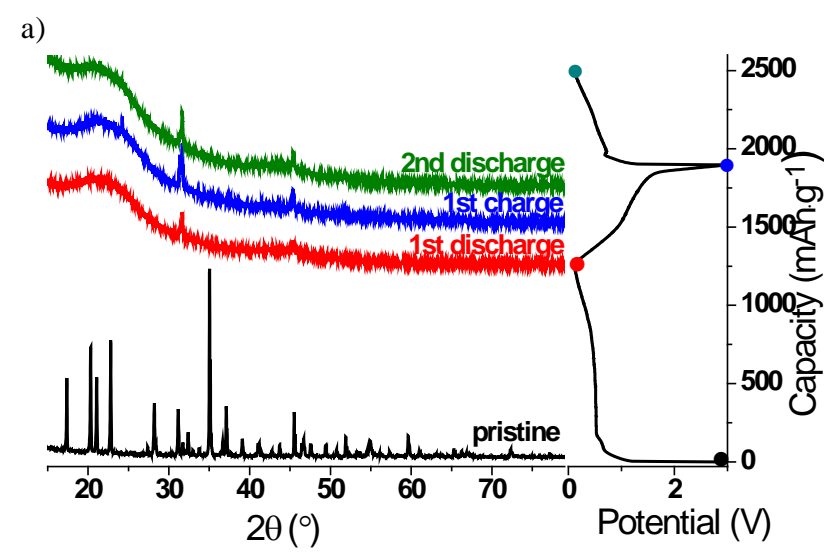

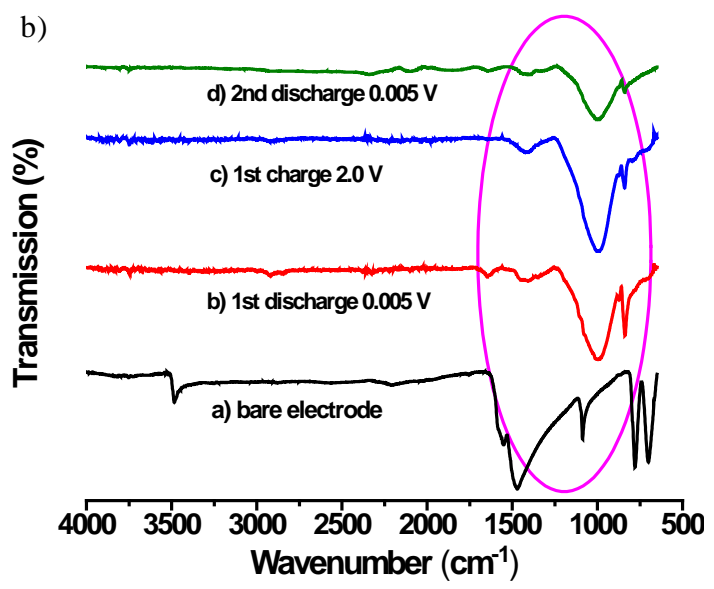

Fig.3. a) PXRD patterns with corresponding discharge and charge curves; b) FT-IR spectra of Mn-MOF under different voltages.

\section{Conclusion}

The squarate based MOF, $\mathrm{Mn}_{2}(\mathrm{OH})_{2}\left(\mathrm{C}_{4} \mathrm{O}_{4}\right)$, with high crystallinity was synthesized through a hydrothermal method and used as anodes in the LIBs. As an anode material for lithium-ion batteries, it delivered a relatively high irreversible capacity in the first discharge process and a reversible lithium storage capacity up to ca. $450 \mathrm{mAh} \mathrm{g}^{-1}$ at the fifty cyclesunder high current density (400 mA $\left.\mathrm{g}^{-1}\right)$. The reversible capacity of Mn-MOF exhibits almost no significant fading after 20 cycles, which indicates its excellent electrochemical performance. Further investigation in terms of the inner electrochemical mechanism is under way.

\section{Acknowledgements}

Financial support for this research was provided by the National Key Technology R\&D Program (2014BAA07B04), China, Development Fund (ZD-15-TYK10), China Postdoctoral Science Foundation (2015M570849) and Shaanxi Province Postdoctoral Scientific Research Foundation in 2015, and is greatly appreciated.

\section{References}

[1] A. Morozan and F. Jaouen, Metal organic frameworks for electrochemical applications, Energy Environ. Sci. 5 (2012) 9269-9290.

[2] M.S.Whittingham, Electrical Energy Storage and Intercalation Chemistry,Science192(1967)1126-1127.

[3] S.-L. Li and Q. Xu, Metal-organic frameworks as platforms for clean energy, Energy Environ. Sci.6(2013) 1656-1683.

[4] Q.-L. Zhu and Q. Xu, Metal-organic framework composites, Chem. Soc. Rev.43(2014) 5468-5512.

[5] Y.-R. Lee, J. Kim, and W.-S. Ahn, Synthesis of metal-organic frameworks: A mini review,Korean J. Chem. Eng. 30(2013) 1667-1680.

[6] Z.-J. Lin, J. Lü, M. Hong, and R. Cao, Metal-organic frameworks based on flexible ligands (FL-MOFs): structures and applications,Chem. Soc. Rev. 43(2014) 5867-5895.

[7] S. Qiu, M. Xue, and G. Zhu, Metal-organic framework membranes: from synthesis to separation application, Chem. Soc. Rev. 43(2014) 6116-6140. 
[8] Y. Cui, Y. Yue, G. Qian, and B. Chen, Luminescent Functional Metal-Organic Frameworks, Chem. Rev.112 (2012) 1126-1162.

[9] H. Furukawa, K. E. Cordova, M. O’Keeffe, and O. M. Yaghi, The chemistry and applications of metal-organic frameworks, Science341(2013) 1230444.

[10]G. Férey, F. Millange, M. Morcrette, C. Serre, M. L. Doublet, J. M. Grenèche, and J. M. Tarascon, Mixed-valence Li/Fe-based metal-organic frameworks with both reversible redox and sorption properties,Angew. Chem. Int. Ed. 46(2007) 3259-3263.

[11] K. Saravanan, M. Nagarathinam, P. Balaya, and J. J. Vittal, Lithium storage in a metal organic framework with diamondoid topology - a case study on metal formates, J. Mater. Chem. 20(2010) 8329-8335.

[12] X. Li, F. Cheng, S. Zhang, and J. Chen, Shape-controlled synthesis and lithium-storage study of metal-organic frameworksZn $n_{4} \mathrm{O}(1,3,5 \text {-benzenetribenzoate) })_{2}, \mathrm{~J}$. Power Sources160(2006) 542-547.

[13] C. Combelles, M. Ben Yahia, L. Pedesseau, and M.-L. Doublet, Design of Electrode Materials for Lithium-Ion Batteries: The Example of Metal-Organic Frameworks,J. Phys. Chem.114(2010) 9518-9527.

[14] Z.-M. Xue, B. Liu, and C.-H. Chen, A theoretical study of electrical and electrochemical properties of dicyanomethylene derivatives of squaric acid, Electrochim. Acta. 51(2006) 4554-4561.

[15] L. Wang, Z. Schnepp, and M. M. Titirici, Rice husk-derived carbon anodes for lithium ion batteries,J. Mater. Chem.1(2013) 5269-5273. 